\title{
Engineering thiol-ene click chemistry for the fabrication of novel structurally well-defined multifunctional cyclodextrin separation materials for enhanced enantioseparation
}

\author{
Xiaobin Yao ${ }^{1,2}$, Hao Zheng ${ }^{3}$, Yang Zhang ${ }^{4}$, Xiaofei Ma ${ }^{1,2}$, Yin Xiao ${ }^{* 2,3}$, Yong Wang ${ }^{* 1,2}$ \\ ${ }^{1}$ Department of Chemistry, School of Science, Tianjin University, Tianjin 300072, China. \\ ${ }^{2}$ Collaborative Innovation Center of Chemical Science and Engineering (Tianjin), Tianjin 300072, \\ China. \\ ${ }^{3}$ School of Chemical Engineering, Tianjin University, Tianjin 300072, China \\ ${ }^{4}$ Weifang Teda Environmental Protection Equipment Co., Ltd, Weifang 262100, China. \\ E-mailaddresses: xiaoyin@tju.edu.cn,wangyongtju@tju.edu.cn.
}

\section{Table of Contents}

Figure S-1 Structures of analytes

Figure S-2 SEM image of thiol silica (a), CSP1 (b) and CSP2 (c)

Figure S-3 TGA analysis of Thiol-silica, CSP1 and CSP2

Figure S-4 The standard curve of quantitative analyses for 4MOPh-OPr (top) and the relationship between chromatographic parameters and sample concentration (bottom) on CSP1 (left) and CSP2 (right)

Figure S-5 Enantioseparation of 4MOPh-OPr under different temperatures (flow rate $=1.0$ $\left.\mathrm{mL} \cdot \mathrm{min}^{-1}\right)$ and flow rates $\left(\mathrm{T}=30^{\circ} \mathrm{C}\right)$ on current $\operatorname{CSP} 1(\mathrm{a}, \mathrm{c})$ and $\operatorname{CSP} 2(\mathrm{~b}, \mathrm{~d})$

Figure S-6 Representative enantioseparation chromatograms of selected analytes. Conditions: flow rate $1 \mathrm{~mL} \cdot \mathrm{min}^{-1} ; 30^{\circ} \mathrm{C}$

Figure S-7 Comparison of CSP1 and CSP2 in PO mode. Conditions: IPA=100\%; flow rate 1 $\mathrm{mL} \cdot \min ^{-1} ; 30{ }^{\circ} \mathrm{C}$

Figure S-8 Reproducibility of CSP1 and CSP2

Table S-1 Elemental analysis of thiol silica and CSPs

Table S-2 Chiral separation of 4MOPh-OPr with ranges of concentrations on CSP1

Table S-3 Chiral separation of 4MOPh-OPr with ranges of concentrations on CSP2

Table S-4 The impact of organic solvent type on separation of isoxazoline derivatives on CSP1

Table S-5 Separation results of analytes with MP at high proportion of organic modifier 


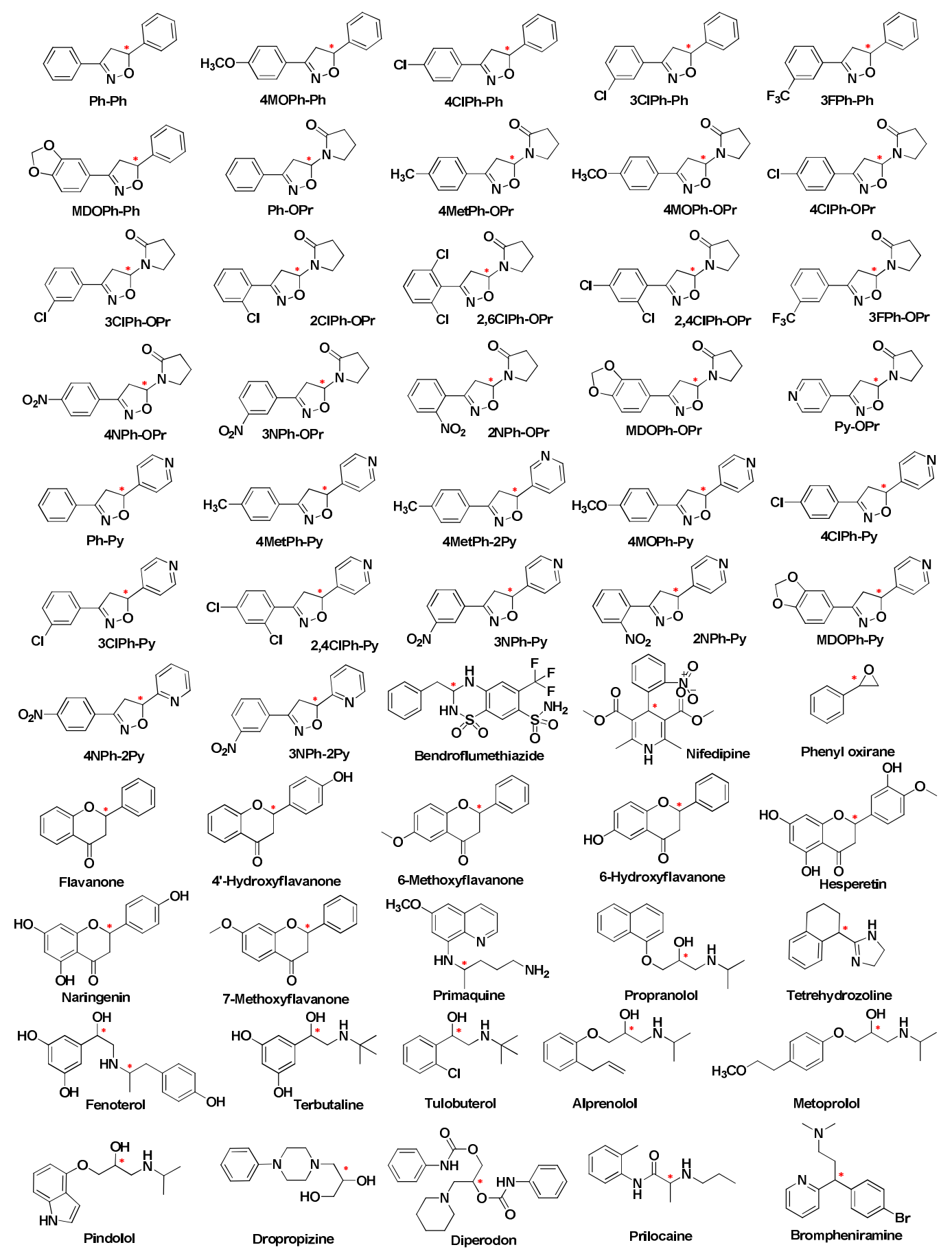

Figure S-1 Structures of analytes 

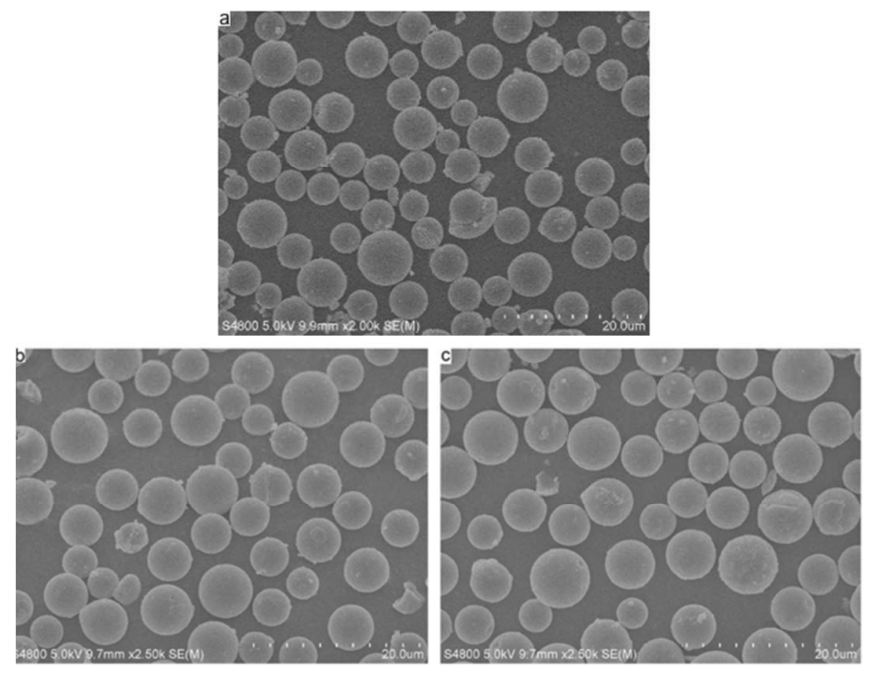

Figure S-2 SEM image of thiol silica (a), CSP1 (b) and CSP2 (c)

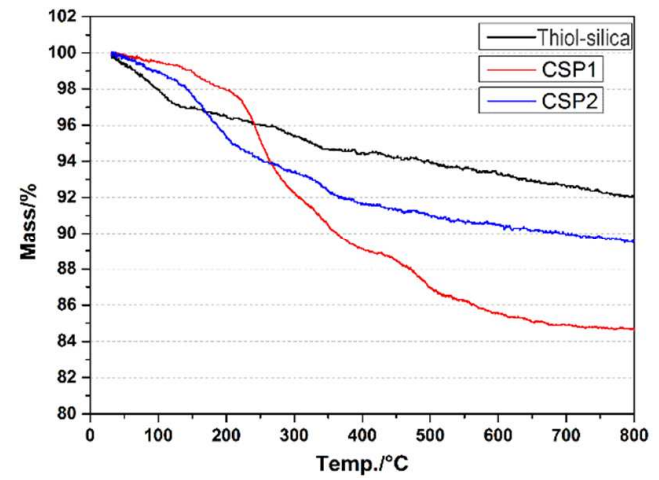

Figure S-3 TGA analysis of Thiol-silica, CSP1 and CSP2 

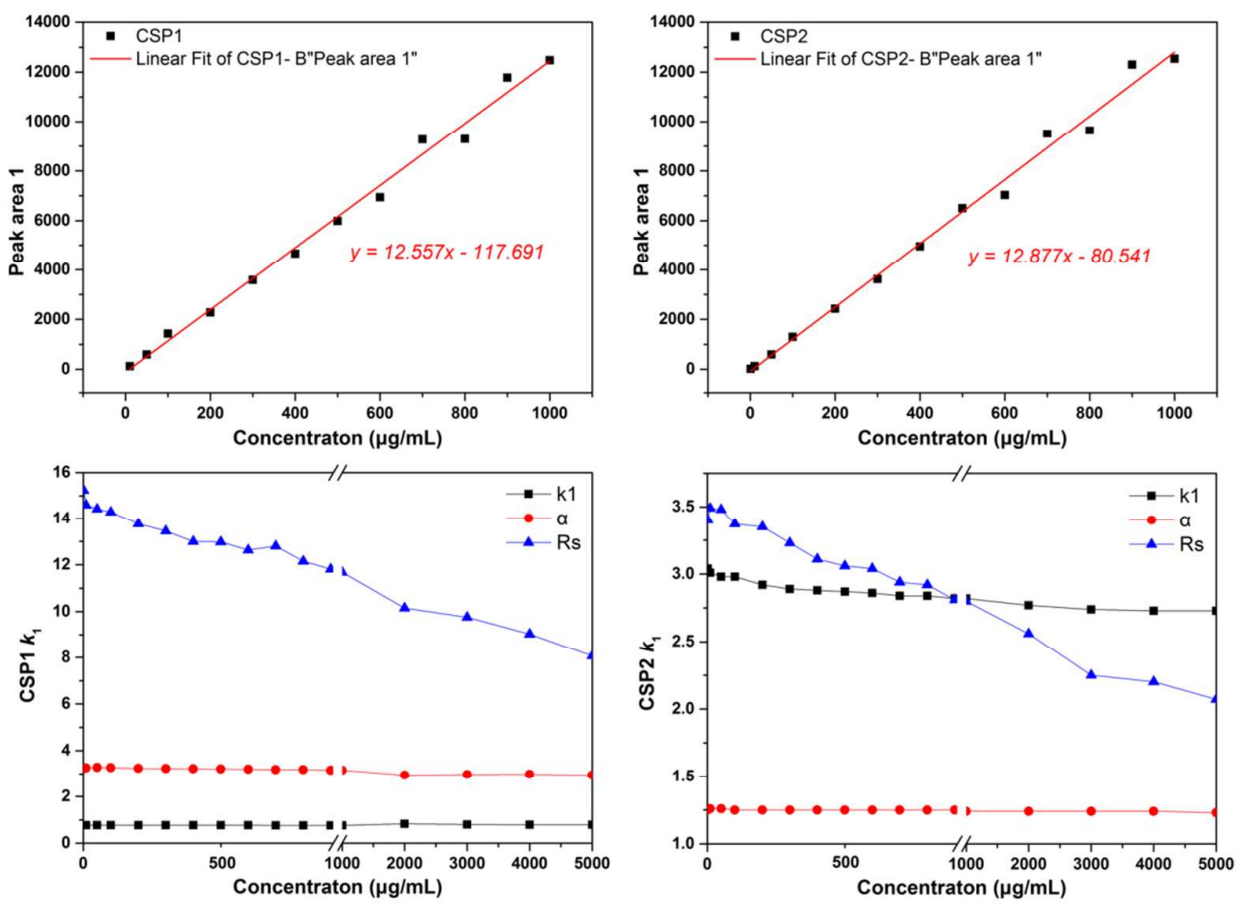

Figure S-4 The standard curve of quantitative analyses for 4MOPh-OPr (top) and the relationship between chromatographic parameters and sample concentration (bottom) on CSP1 (left) and CSP2 (right)
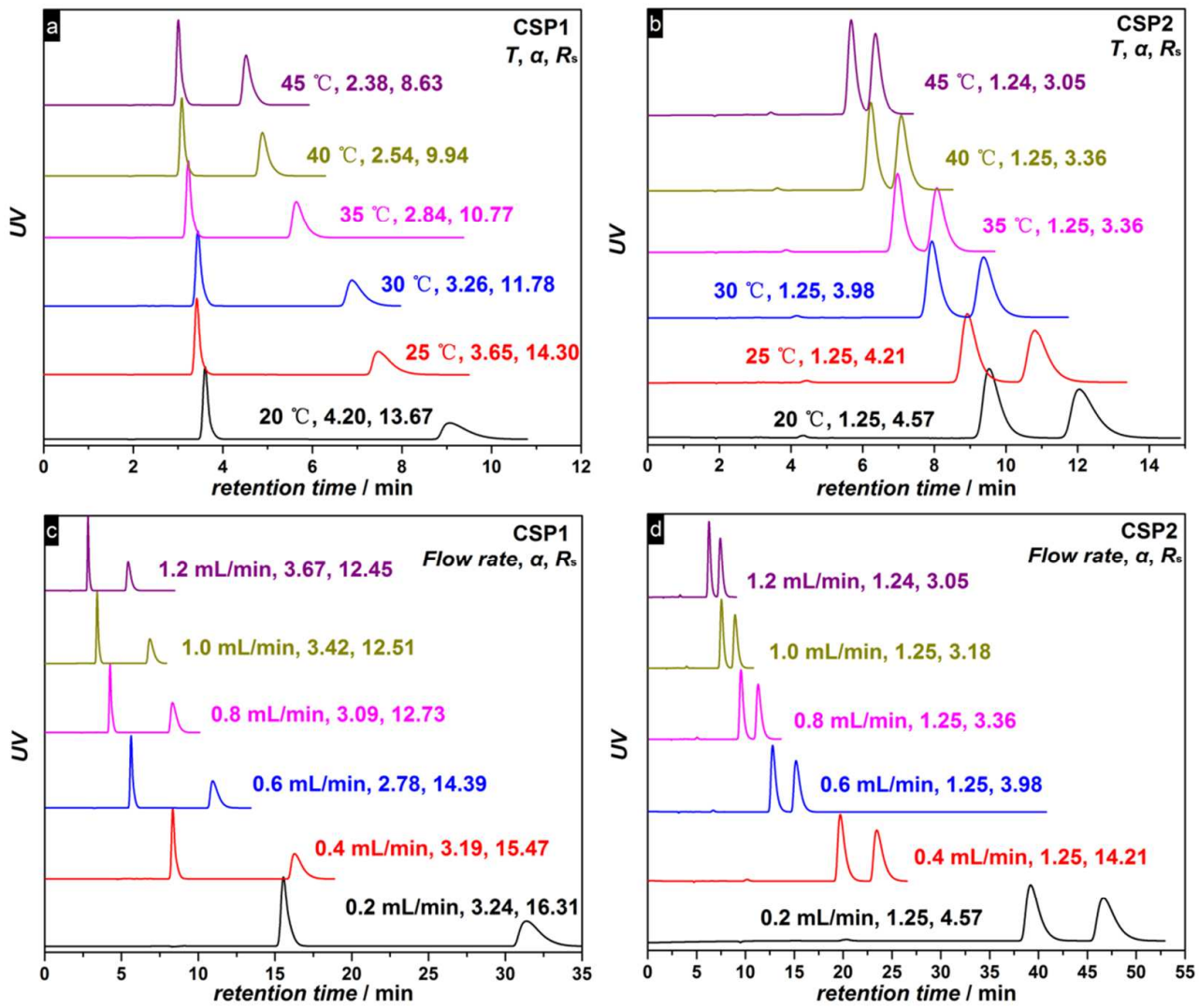

Figure S-5 Enantioseparation of 4MOPh-OPr under different temperatures (flow rate $=1.0 \mathrm{~mL} \cdot \mathrm{min}^{-1}$ ) and flow rates $\left(\mathrm{T}=30^{\circ} \mathrm{C}\right)$ on current $\operatorname{CSP} 1(\mathrm{a}, \mathrm{c})$ and $\operatorname{CSP} 2(\mathrm{~b}, \mathrm{~d})$ 

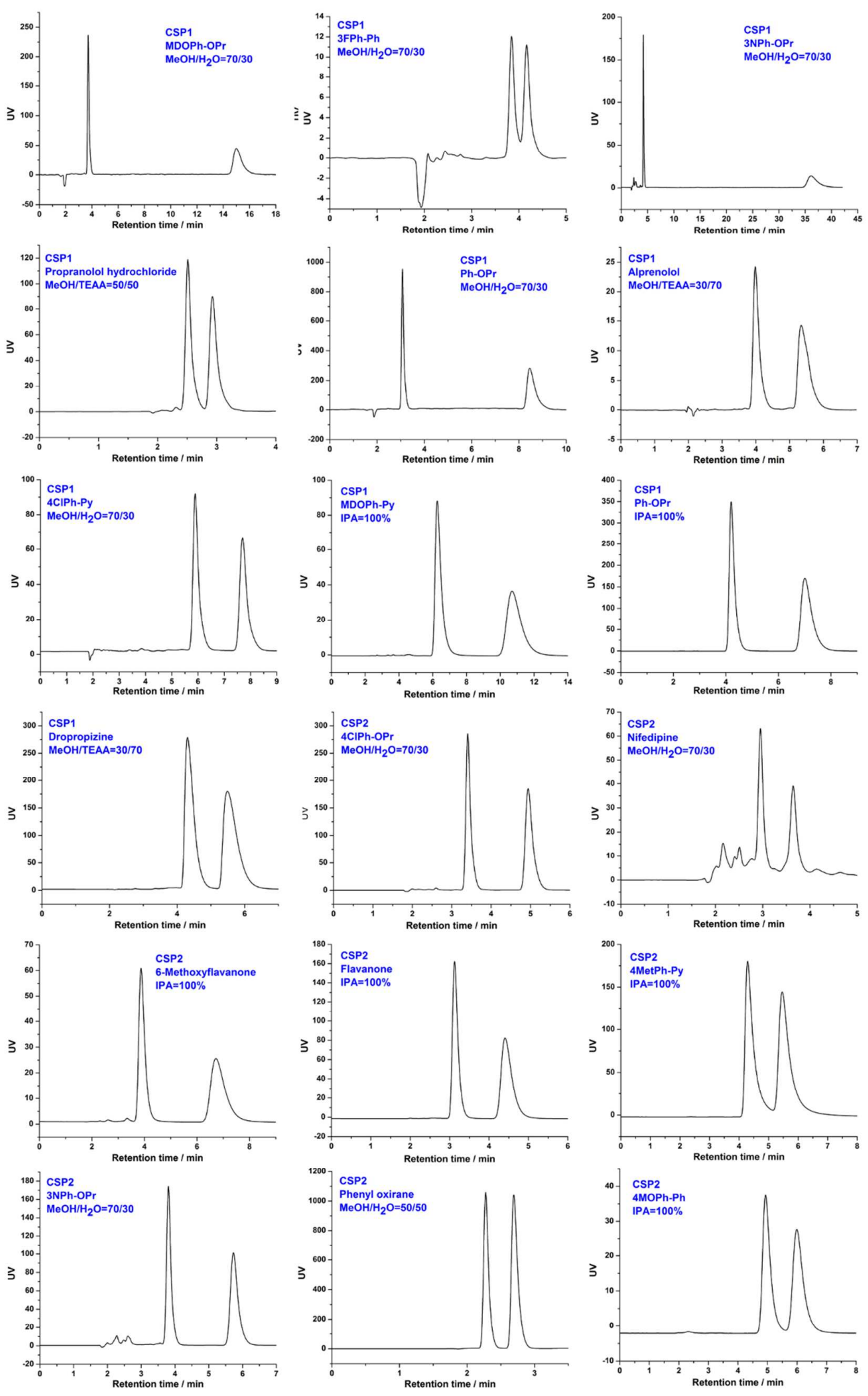

Figure S-6 Representative enantioseparation chromatograms of selected analytes

Conditions: flow rate $1 \mathrm{~mL} \cdot \mathrm{min}^{-1} ; 30{ }^{\circ} \mathrm{C}$ 

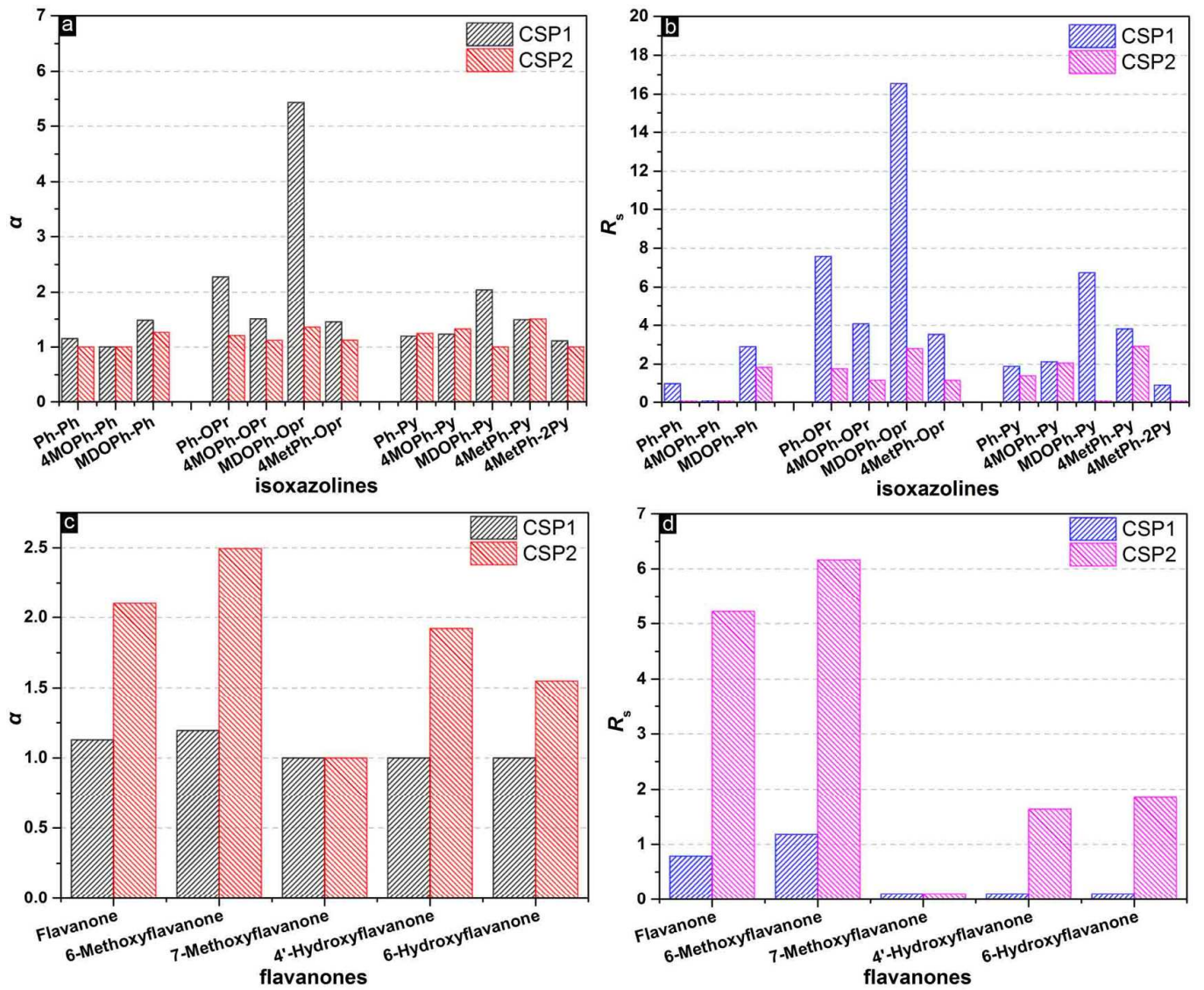

Figure S-7 Comparison of CSP1 and CSP2 in PO mode.

Conditions: IPA $=100 \%$; flow rate $1 \mathrm{~mL} \cdot \mathrm{min}^{-1} ; 30{ }^{\circ} \mathrm{C}$. 

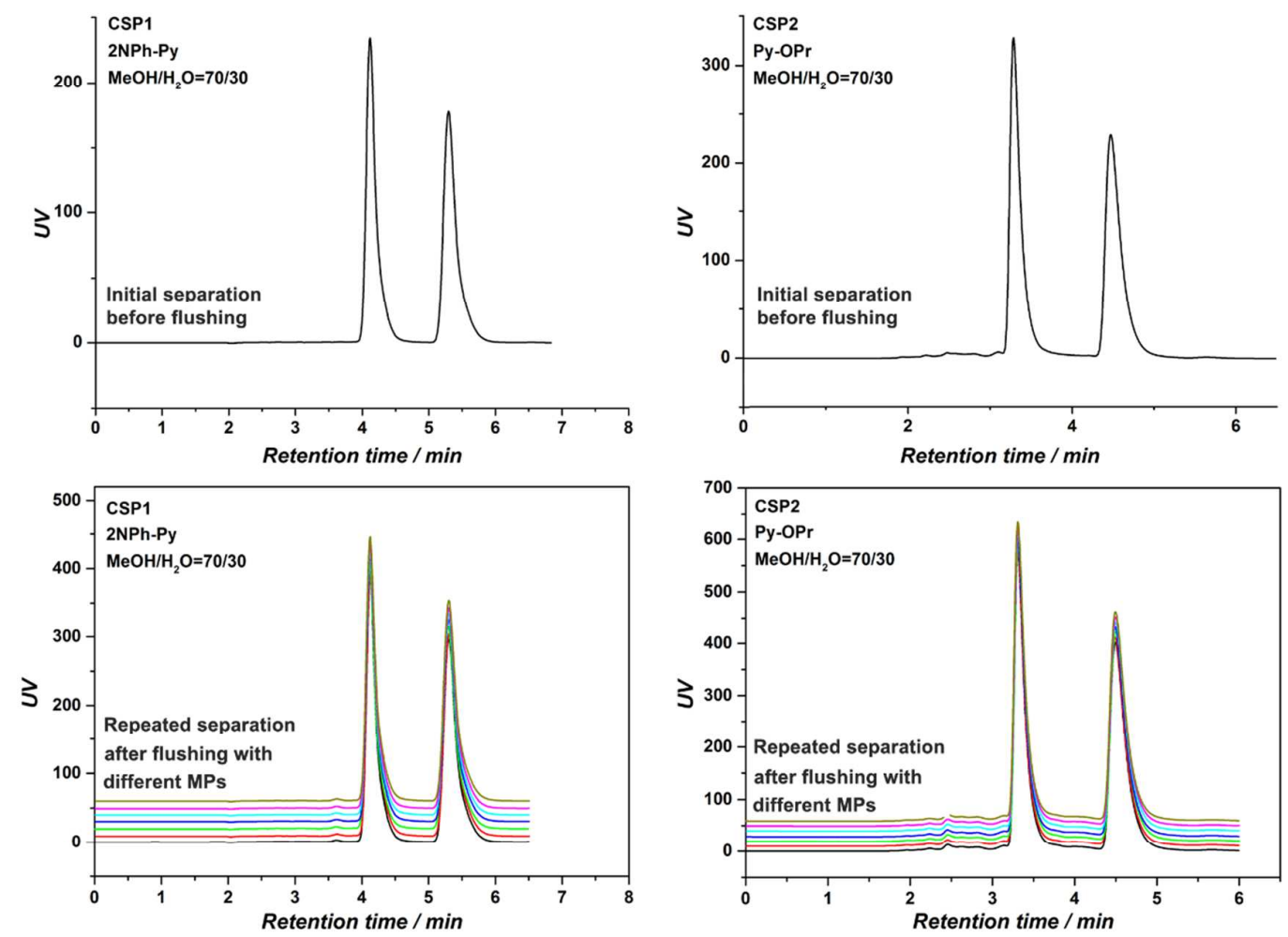

Figure S-8 Reproducibility of CSP1 and CSP2

Table S-1 Elemental analysis of thiol silica and CSPs

\begin{tabular}{ccccc}
\hline Analytes & $\mathrm{C}(\%)$ & $\mathrm{H}(\%)$ & $\mathrm{N}(\%)$ & $\mathrm{S}(\%)$ \\
\hline Thiol silica & 1.79 & 1.43 & 0.46 & 0.71 \\
CSP1 & 8.10 & 1.94 & 0.68 & 0.65 \\
CSP2 & 5.46 & 1.66 & 0.70 & 0.68 \\
\hline
\end{tabular}

Table S-2 Chiral separation of 4MOPh-OPr with ranges of concentrations on CSP1

\begin{tabular}{ccccccc}
\hline $\begin{array}{c}\text { Concentration } \\
/(\mu \mathrm{g} / \mathrm{mL})\end{array}$ & $k_{1}$ & $k_{2}$ & $\alpha$ & $R_{s}$ & Peak area 1 & Peak area 2 \\
\hline 1 & 0.77 & 2.50 & 3.23 & 15.23 & 17 & 17 \\
10 & 0.77 & 2.51 & 3.26 & 14.59 & 120 & 116 \\
50 & 0.77 & 2.53 & 3.28 & 14.42 & 594 & 592 \\
100 & 0.77 & 2.51 & 3.27 & 14.29 & 1425 & 1429 \\
200 & 0.77 & 2.49 & 3.24 & 13.76 & 2280 & 2285 \\
300 & 0.77 & 2.49 & 3.23 & 13.45 & 3579 & 3567 \\
400 & 0.77 & 2.47 & 3.22 & 13.00 & 4670 & 4688 \\
500 & 0.77 & 2.46 & 3.21 & 12.98 & 5984 & 6103 \\
600 & 0.77 & 2.45 & 3.20 & 12.63 & 6939 & 7075 \\
700 & 0.76 & 2.43 & 3.18 & 12.80 & 9274 & 9418 \\
\hline
\end{tabular}




\begin{tabular}{ccccccc}
800 & 0.76 & 2.43 & 3.18 & 12.15 & 9288 & 9548 \\
900 & 0.76 & 2.41 & 3.16 & 11.81 & 11774 & 12121 \\
1000 & 0.76 & 2.41 & 3.16 & 11.68 & 12477 & 12927 \\
2000 & 0.83 & 2.43 & 2.94 & 10.15 & 18893 & 21021 \\
3000 & 0.80 & 2.38 & 2.97 & 9.75 & 21749 & 25818 \\
4000 & 0.79 & 2.35 & 2.98 & 9.03 & 26259 & 34249 \\
5000 & 0.79 & 2.33 & 2.94 & 8.07 & 30983 & 45796 \\
\hline
\end{tabular}

\begin{tabular}{ccccccc}
\multicolumn{6}{l}{ Table S-3 Chiral separation of 4MOPh-OPr with ranges of concentrations on CSP2 } \\
\hline $\begin{array}{c}\text { Concentration/ } \\
(\mu \mathrm{g} / \mathrm{mL})\end{array}$ & $k_{1}$ & $k_{2}$ & $\alpha$ & $R_{s}$ & Peak area 1 & Peak area 2 \\
\hline 1 & 3.04 & 3.80 & 1.25 & 3.40 & 18 & 17 \\
10 & 3.01 & 3.79 & 1.26 & 3.49 & 121 & 119 \\
50 & 2.98 & 3.74 & 1.26 & 3.48 & 595 & 608 \\
100 & 2.98 & 3.74 & 1.25 & 3.37 & 1296 & 1297 \\
200 & 2.92 & 3.66 & 1.25 & 3.35 & 2431 & 2446 \\
300 & 2.89 & 3.63 & 1.25 & 3.23 & 3618 & 3686 \\
400 & 2.88 & 3.60 & 1.25 & 3.11 & 4964 & 4972 \\
500 & 2.87 & 3.58 & 1.25 & 3.06 & 6503 & 6578 \\
600 & 2.86 & 3.57 & 1.25 & 3.04 & 7029 & 7169 \\
700 & 2.84 & 3.54 & 1.25 & 2.94 & 9509 & 9621 \\
800 & 2.84 & 3.54 & 1.25 & 2.92 & 9645 & 9815 \\
900 & 2.82 & 3.51 & 1.25 & 2.81 & 12299 & 12528 \\
1000 & 2.82 & 3.50 & 1.24 & 2.80 & 12534 & 12714 \\
2000 & 2.77 & 3.44 & 1.24 & 2.56 & 21100 & 21578 \\
3000 & 2.74 & 3.40 & 1.24 & 2.25 & 31569 & 193132 \\
4000 & 2.73 & 3.38 & 1.24 & 2.20 & 43409 & 44943 \\
5000 & 2.73 & 3.37 & 1.23 & 2.07 & 51607 & 54011 \\
\hline
\end{tabular}

Table S-4 The impact of organic solvent type on separation of isoxazoline derivatives on CSP 1

\begin{tabular}{|c|c|c|c|c|c|}
\hline analytes & $k_{1}$ & $k_{2}$ & $\alpha$ & $R_{\mathrm{s}}$ & $\mathrm{MP}(\mathrm{V} / \mathrm{V})$ \\
\hline $\mathrm{Ph}-\mathrm{Ph}$ & 0.93 & 1.03 & 1.11 & 1.50 & \multirow{4}{*}{$\mathrm{MeOH} / \mathrm{H}_{2} \mathrm{O}=70 / 30$} \\
\hline $\mathrm{Ph}-\mathrm{OPr}$ & 0.63 & 3.48 & 5.50 & 19.61 & \\
\hline 4ClPh-Ph & 1.83 & 2.51 & 1.37 & 6.34 & \\
\hline 4ClPh-Py & 2.12 & 3.07 & 1.45 & 6.46 & \\
\hline $\mathrm{Ph}-\mathrm{Ph}$ & 15.82 & - & 1 & 0 & \multirow{4}{*}{$\mathrm{ACN} / \mathrm{H}_{2} \mathrm{O}=30 / 70$} \\
\hline $\mathrm{Ph}-\mathrm{OPr}$ & 2.12 & 2.27 & 1.07 & 1.41 & \\
\hline 4ClPh-Ph & 4.30 & 4.46 & 1.04 & 0.98 & \\
\hline 4ClPh-Py & 7.99 & 8.39 & 1.05 & 1.51 & \\
\hline
\end{tabular}

Conditions: flow rate $1 \mathrm{~mL} \cdot \mathrm{min}^{-1} ; 30{ }^{\circ} \mathrm{C}$. 
Table S-5 Separation results of analytes with MP at high proportion of organic modifier

\begin{tabular}{lccccc}
\hline analytes & $k_{1}$ & $k_{2}$ & $\alpha$ & $R_{\mathrm{s}}$ & $\mathrm{MeOH} / \mathrm{H}_{2} \mathrm{O}(\mathrm{V} / \mathrm{V})$ \\
\hline \multirow{3}{*}{ Ph-OPr } & 0.37 & 2.66 & 7.29 & 19.14 & $80 / 20$ \\
& 0.23 & 2.35 & 10.16 & 21.17 & $90 / 10$ \\
& 0.26 & 2.72 & 10.57 & 20.10 & $95 / 5$ \\
& 0.34 & 2.93 & 8.62 & 22.27 & $97.5 / 2.5$ \\
& 0.34 & 2.96 & 8.65 & 19.67 & $99 / 1$ \\
$4 \mathrm{MOPh}-\mathrm{OPr}$ & 0.42 & 1.61 & 3.84 & 13.17 & $80 / 20$ \\
& 0.28 & 1.38 & 4.83 & 11.94 & $90 / 10$ \\
& 0.34 & 1.55 & 4.56 & 12.11 & $95 / 5$ \\
& 0.39 & 1.66 & 4.27 & 9.15 & $97.5 / 2.5$ \\
\hline
\end{tabular}

Conditions: flow rate $1 \mathrm{~mL} \cdot \mathrm{min}^{-1} ; 30{ }^{\circ} \mathrm{C}$. 